\title{
Cone dysfunctions in retinitis pigmentosa with retinal nerve fiber layer thickening
}

This article was published in the following Dove Press journal:

Clinical Ophthalmology

24 March 2012

Number of times this article has been viewed

\section{Güngör Sobacı \\ Gökhan Özge \\ Fatih Ç Gündoğan}

Department of Ophthalmology, Gülhane Military Medical Academy and Medical School, Etlik, Ankara, Turkey
Correspondence: Güngör Sobacı Gülhane Askeri Tıp Akademisi, Göz AD, Etlik, Ankara, 06018, Turkey

Tel 903124171816

Fax +90312417 1816

Email gsobaci@hotmail.com
Purpose: To investigate whether or not thicker retinal nerve fiber layer (RNFL) in retinitis pigmentosa (RP) patients relates to functional abnormalities of the photoreceptors.

Methods: Optical coherence tomography-based RNFL thickness was measured by Stratus-3 ${ }^{\text {TM }}$ (Zeiss, Basel, Switzerland) optical coherence tomography and electroretinogram (ERG) recordings made using the RETI-port ${ }^{\circledR}$ system (Roland, Wiesbaden, Germany) in 27 patients with retinitis pigmentosa and in 30 healthy subjects.

Results: Photopic ERG b-wave amplitude, cone ERG b-wave latency, $30 \mathrm{~Hz}$ flicker amplitude, and $30 \mathrm{~Hz}$ flicker latency had significant correlations to the RNFL-temporal $(r=-0.55$, $P=0.004, r=0.68, P=0.001, r=-0.65, P=0.001$, and $r=-0.52, P=0.007$, respectively). Eyes with thicker RNFL (ten eyes) differed significantly from those with thinner RNFL (eight eyes) regarding cone ERG b-wave latency values only $(P=0.001)$.

Conclusion: Thicker RNFL in patients with retinitis pigmentosa may be associated with functional abnormality of the cone system.

Keywords: optical coherence tomography, electroretinography, photoreceptor, b-wave

\section{Introduction}

Retinitis pigmentosa (RP) is one of the most common forms of hereditary retinal degeneration. It is characterized by the progressive loss of outer retinal function and may eventually lead to blindness, which is incurable. Integrity of the inner retinal layer has been suggested as a prerequisite for successful replacement/restoration therapy in the outer retinal layer (ORL). Optical coherence tomography (OCT) providing highresolution, cross-sectional images which correspond to histological sections can be used in evaluation of the retinal nerve fiber layer (RNFL). ${ }^{1}$ Recently, variability in OCT-assessed RNFL thickness in patients with RP has been shown. ${ }^{2-4}$ RNFL thickness as obtained by OCT can be used clinically to assess the integrity of the inner retinal layer in RP. Among the sectors analyzed by OCT, the RNFL-temporal sector, consisting of maculopapular fibers, has elicited special interest since it conveys visual information from the macula to the lateral geniculate nucleus.

In light of recent findings, ${ }^{5,6}$ which highlight transsynaptic anterograde and retrograde neural degeneration in the retina, we hypothesize that thicker RNFL in RP may be related to functional abnormalities of the photoreceptors in the ORL.

\section{Materials and methods}

Twenty-seven patients with RP (group 1) and 30 healthy subjects (group 2) were enrolled. This study adheres to the tenets of the Declaration of Helsinki (as revised in 
Edinburgh 2000) $;^{7}$ informed consent was obtained from the subjects, and patient anonymity was preserved.

They were selected among conscripts between the ages of 20 to 27 (mean: $23.1 \pm 2.4$ ) years old. Only one of the eyes with better visual acuity (VA) in the groups was studied. Patients who had VA of more than 1 logMAR (less than 20/200 Snellen equivalent), refractive errors with spherical equivalent of more than 3 diopters, large or small optic discs, intraocular pressure and visual fields suggesting glaucoma, and cystoid macular edema were excluded. Electroretinogram (ERG) recordings (RETI-port ${ }^{\circledR}$, Roland, Wiesbaden, Germany) according to the International Society for Clinical Electrophysiology of Vision standards, ${ }^{8}$ and RNFL measurements using the fast RNFL algorithm (Stratus-3 ${ }^{\mathrm{TM}}$, Zeiss, Basel, Switzerland) were performed in the groups. With the fast RNFL thickness protocol, three 3.4-mm diameter circular scans were acquired. Comparison to an age-matched normative database is performed automatically by the OCT for overall RNFL thickness and the quadrants (superior: 46 to 135 degrees; nasal: 136 to 225 degrees; inferior: 226 to 315 degrees, and temporal: 316 to 45 degrees), and RNFL thickness in twelve 30-degree clock-hour sectors). In addition, central macular thickness (CMT) was analyzed. CMT was referred to the central 1-mm zone of the macular thickness map. The fast macular thickness map scan protocol on the OCT3 (Stratus ${ }^{\mathrm{TM}}$ ) was used by the same trained operator. Only complete, well-centered scans with signal strengths of more than 6 and correct segmentation were used. A mean of three successive measurements was used for statistical analysis. RNFL and CMT in group 1 were evaluated regarding normal values in controls (group 2); those outside 5\%-95\% confidence interval limits.

All measurements were undertaken by the same examiner in the morning, and an average of three successive measurements were analyzed using SPSS software (v 13.0; IBM, Armonk, NY). Correlations of VAs as logMAR-VA to RNFL thickness, VAs to ERG parameters, and RNFL thickness to ERG parameters in the RP group were analyzed by Spearman test; Student's $t$-test, and the Mann-Whitney $U$ test for comparing means, and Fisher's exact test for associations between variables. A $P$ value of $<0.01$ was considered statistically significant in statistical analyses.

\section{Results}

The mean CMT value did not differ between the groups $(215 \pm$ $25 \mu \mathrm{m}$ and $212 \pm 28 \mu \mathrm{m} ; P=0.67$, Student's $t$-test). All RNFL thickness values in the control group were within the normal limits of the OCT3-based normative database. Ten eyes (37\%) in the RP group had thicker RNFL ( $>95 \%$ confidence interval upper limits from the control group) than controls. These thicker RNFL values were distributed in different sectors either separately or side by side around the optic disk (Table 1). Nine of ten eyes with thicker RNFL were located in the temporal quadrant, and one (10\%) in the superior quadrant. Nine eyes had normal RNFL values in all four quadrants. Eight (29.6\%) eyes in the RP group had thinner RNFL values in addition to normal values. There was no difference in the distribution of thinner RNFL in the quadrants; however, significant differences existed between RNFL-temporal and RNFL-nasal and RNFL inferior ( $P=0.019$ for both, Fisher's exact test) in regard to thickening in RNFL. Table 2 gives correlation coefficients between RNFL-overall and ERG parameters, and RNFL-temporal and ERG parameters in the RP group. Cone ERG b-wave latency and $30 \mathrm{~Hz}$ flicker amplitude showed significant correlations to the RNFL-overall $(r=-0.60$, $P=0.001$ and $r=-0.51, P=0.008$, respectively) (Figure 1 and Table 2). Photopic ERG b-wave amplitude, cone ERG b-wave latency, $30 \mathrm{~Hz}$ flicker amplitude, and $30 \mathrm{~Hz}$ flicker latency had significant correlations to the RNFL-temporal $(r=-0.55, P=0.004, r=0.68, P=0.001, r=-0.65, P=0.001$, and $r=-0.52, P=0.007$, respectively) (Table 2 ). The mean of thicker RNFL was 55\% more than the mean of normal RNFL thickness. Eyes with thicker RNFL (ten eyes) differed significantly from those with thinner ones (eight eyes) regarding cone ERG b-wave latency values ( $P=0.001$, Mann-Whitney $U$ test) only. Figure 2 shows the scatterplot of the relationship between the cone ERG b-wave and RNFL-temporal ( $r=0.68$, $P=0.001)$. Insignificant correlations were found between VAs and both RNFL-overall ( $r=-0.22, P=0.36$, Spearman test) and RNFL-temporal ( $r=-0.23, P=0.35$, Spearman test) values in the RP group. No significant correlation was found between VAs and ERG parameters in the RP group $(P>0.01$ for all, Spearman test).

\section{Discussion}

We observed both thicker (37\%) and thinner (29.6\%) RNFL values, which is not an unexpected finding in

Table I Distribution of abnormal segments in various quadrants in patients with retinitis pigmentosa $(n=27)$

\begin{tabular}{llll}
\hline Quadrants & Thicker RNFL & Normal & Thinner \\
\cline { 2 - 4 } & $\mathbf{n}(\%)$ & $\mathbf{n}(\%)$ & $\mathbf{n}(\%)$ \\
\hline Nasal & $2(7 \%)$ & $21(78 \%)$ & $4(15 \%)$ \\
Inferior & $2(\% 7)$ & $21(78 \%)$ & $4(15 \%)$ \\
Temporal & $9(33 \%)$ & $15(56 \%)$ & $2(7 \%)$ \\
Superior & $4(15 \%)$ & $17(63 \%)$ & $6(22 \%)$ \\
\hline
\end{tabular}

Abbreviation: RNFL, retinal nerve fiber layer. 
Table 2 Correlations between retinal nerve fiber layer thickness and electroretinogram parameters

\begin{tabular}{|c|c|c|c|c|c|c|c|c|c|}
\hline \multirow{2}{*}{$\begin{array}{l}\text { ERG } \\
\text { parameters }\end{array}$} & \multicolumn{2}{|l|}{ Scotopic } & \multicolumn{2}{|l|}{ Photopic } & \multirow{2}{*}{$\frac{\text { Oss }}{\mathrm{PI}}$} & \multicolumn{2}{|l|}{ Cone } & \multicolumn{2}{|l|}{$30 \mathrm{~Hz}$} \\
\hline & $b(a m p)$ & b(lat) & $b(a m p)$ & b(lat) & & b(amp) & b(lat) & amp & lat \\
\hline \multirow[t]{2}{*}{ RNFL-overall } & $r=-0.27$ & $r=-0.07$ & $r=-0.33$ & $r=0.01$ & $r=0.14$ & $r=0.34$ & $r=-0.60$ & $r=-0.5 \mathrm{I}$ & $r=0.34$ \\
\hline & $P=0.18$ & $P=0.73$ & $P=0.10$ & $P=0.94$ & $P=0.77$ & $P=0.09$ & $P=0.001$ & $P=0.008$ & $P=0.09$ \\
\hline \multirow[t]{2}{*}{ RNFL-temp } & $r=-0.37$ & $r=-0.29$ & $r=-0.55$ & $r=0.08$ & $r=-0.15$ & $r=-0.33$ & $r=0.46$ & $r=-0.65$ & $r=-0.57$ \\
\hline & $P=0.06$ & $P=0.15$ & $P=0.004$ & $P=0.69$ & $P=0.45$ & $P=0.10$ & $P=-0.01$ & $P=0.001$ & $P=0.003$ \\
\hline
\end{tabular}

Abbreviations: RNFL, retinal nerve fiber layer; ERG, electroretinogram; Oss, ossilatory potentials.

RP patients. ${ }^{1-4}$ Regarding transneuronal ganglion cell death, exact cause-effect relationship has not yet been clarified in all RP cases. ${ }^{9}$ In addition to transsynaptic degeneration, degeneration of the bipolar, amacrine, and Müller cells may be responsible for variability in RNFL thickness in RP patients. ${ }^{10}$ OCT analysis, which gives invaluable information about the microstructure of the retina, has become an indispensible part of the clinical examination in patients with RP. ERG combined with OCT analysis may reliably reveal the relation between microstructure and function. Oishi et al showed that no correlation existed between RNFL and VA, inheritance trait, laterality, refractive error, or the extent of visual field defect. ${ }^{11}$ They also found ageing and being male were significant risk factors for thinner RNFL. Our study population consisted of only young adult males in both groups. Except for RP in the study group, they had no ocular or systemic disease. They had no structural abnormality in the macula discernible by OCT. We found that almost 30 percent of the patients had thinner RNFL. We found no correlation between VA and RNFL, and VA and ERG parameters. Sugita et al in a focal macular ERG study stated that preserved macular morphology does not necessarily guarantee reserved electrical potential in RP patients. ${ }^{12}$ In a multifocal ERG study, Wolsley et al reported that preserved timing in the central retina, despite significant disruption of the retinal laminar structure, could be suggestive of inner retinal remodeling or functional redundancy. ${ }^{13}$ They also stated that cone-system activity shown in multifocal ERG responses could be related to the thickness of the photoreceptor layer in the macular region.
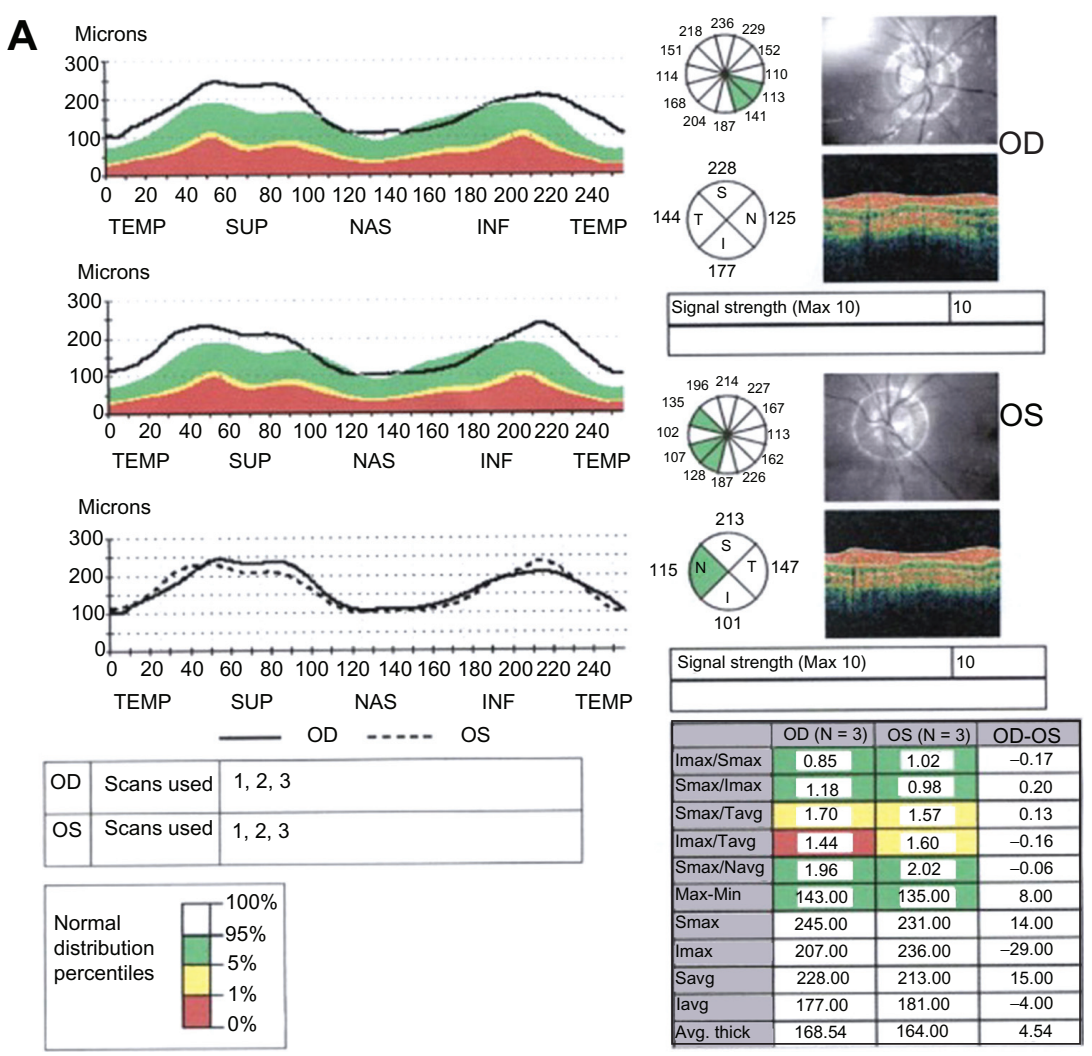

Figure I Retinitis pigmentosa (RP) patient with (A) thicker retinal nerve fiber layer (RNFL) (B) and flash electroretinogram (ERG). 
Figure I (Continued)

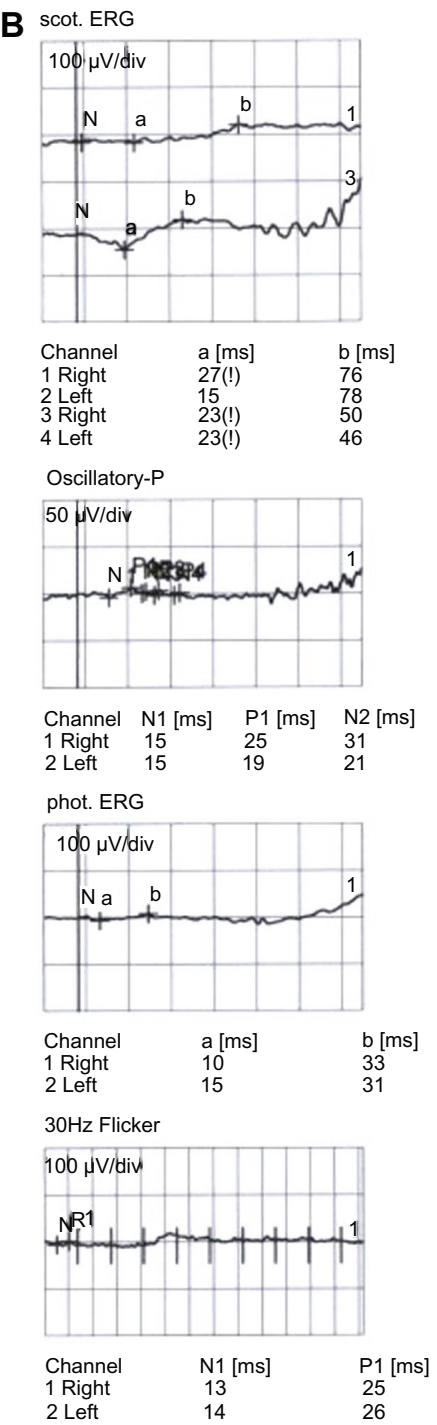

In this study, cone ERG b-wave latency, which showed significant correlation to both RNFL-overall and RNFLtemporal, was significantly delayed in eyes with thicker RNFL values. We showed that eyes with thicker RNFL differed significantly from those with thinner ones in the distribution of this ERG parameter. This correlation may implicate the cause-effect relationship through lack or deficiency of tropic factors from abnormal cones to RNFL in RP patients. We observed no gliotic changes in the inner retinal layer or vitreoretinal interface in the RP group. In the light of findings that highlights transsynsaptic anterograde and retrograde neural degeneration in the retina, ${ }^{5,6,14,15}$ we suggest that some negative (namely, neurotoxic) effect from dysfunctional cones might be the cause of RNFL thickening, which is followed by RNFL thinning later in the course of RP. Unfortunately, the morphological, biochemical, and
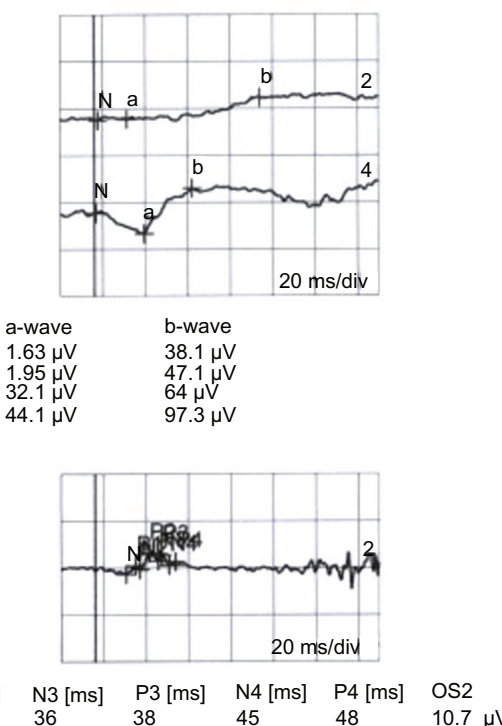
$\begin{array}{lllll}36 & 38 & 45 & 48 & 10.7 \mu \mathrm{V} \\ 30 & 31 & 35 & 38 & 8.95 \mu \mathrm{V}\end{array}$
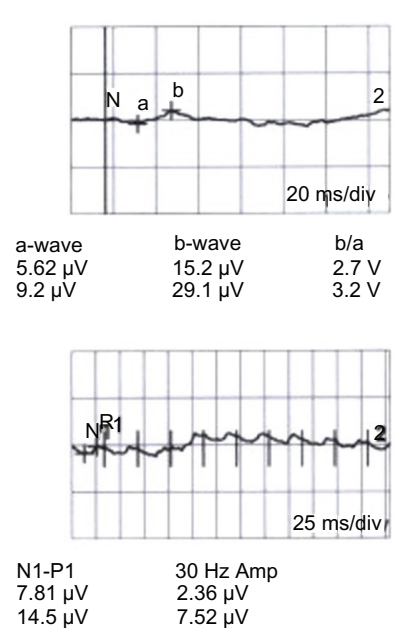

molecular mechanisms involved in the development of RP are not yet fully understood.

The limitations of our study include the following: the numbers of the patients evaluated, and the absence of evaluation of the relationship between genotype and RNFL thickness in RP patients. We applied strict exclusion criteria in this study. All clinical and laboratory studies were performed by the same examiners under the same testing conditions. We have to admit that our findings need to be confirmed by further longitudinal clinical studies with longer follow-up periods in larger groups. Newer, higherresolution spectral OCT techniques might have provided additional information about structural and functional changes in the ORL.

Currently proposed therapies with the application of stem cells, gene deliveries, and retinal implants are based on the 


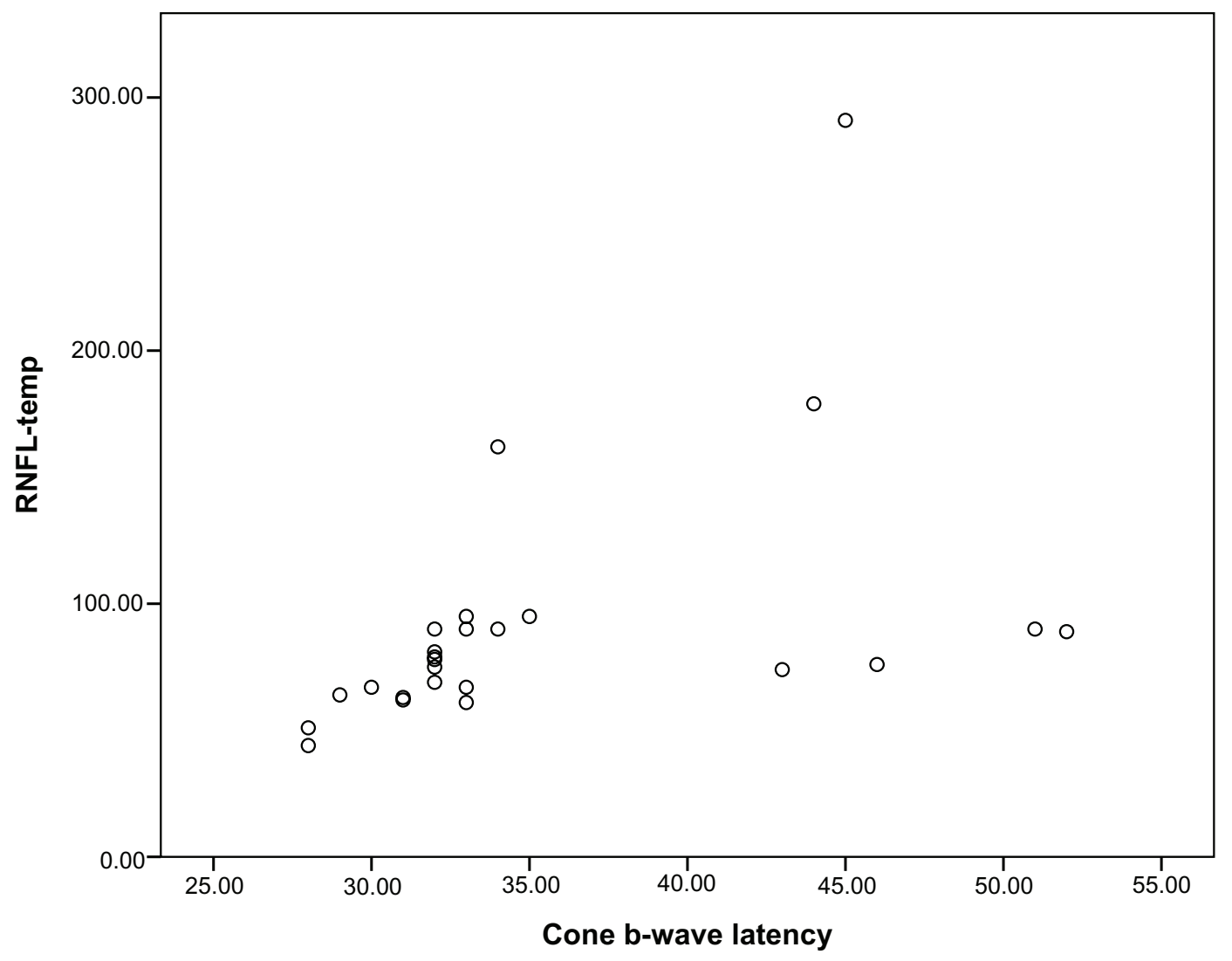

Figure 2 Correlations of cone electroretinogram b-wave latency to thicker retinal nerve fiber layer-temporal thickness.

premise that the inner retinal layer is relatively preserved despite severe destruction in the ORL.

This study shows that thicker RNFL in RP may related to functional abnormalities of the cone system. Further studies are needed to determine the prognostic significance of cone ERG in successful replacement/restoration therapies in RP.

\section{Disclosure}

The authors have no competing/conflicts of interest, and no financial or proprietary interest in this study.

\section{References}

1. Saloni W, Fishman GA, Edward DP, Lindeman M. Retinal nerve fiber layer defects in RP patients. Invest Ophthalmol Vis Sci. 2007;48(10): $4748-4752$.

2. Walia S, Fishman GA. Retinal nerve fiber layer analysis in RP patients using Fourier-domain OCT. Invest Ophthalmol Vis Sci. 2008;49(8):3525-3528.

3. Hood DC, Lin CE, Lazow MA, Locke KG, Zhang X, Birch DG. Thickness of receptor and post-receptor retinal layers in patients with retinitis pigmentosa measured with frequency-domain optical coherence tomography. Invest Ophthalmol Vis Sci. 2009;50(5):2328-2336.

4. Pasadhika S, Fishman GA, Allikmets R, Stone EM. Peripapillary retinal nerve fiber layer thinning in patients with autosomal recessive cone-rod dystrophy. Am J Ophthalmol. 2009;148(2):260-265.

5. YücelY, Gupta N. Glaucoma of the brain: a disease model for the study of transsynaptic neural degeneration. Prog Brain Res. 2008;173:465-478.
6. Bridge $\mathrm{H}$, Jindahra P, Barbur J, Plant GT. Imaging reveals optic tract degeneration in hemianopia. Invest Ophthalmol Vis Sci. 2011;52(1): 382-388.

7. wma.net [homepage on the Internet] World Medical Association. Declaration of Helsinki. Ethical Principles for Medical Research Involving Human Subjects. Available from: http://www.wma.net/ en/30publications/10policies/b3/17c.pdf. Accessed January 26, 2012.

8. Marmor MF, Fulton AB, Holder GE, Miyake Y, Brigell M, Bach M; for International Society for Clinical Electrophysiology of Vision. ISCEV Standard for full-field clinical electroretinography (2008 update). Doc Ophthalmol. 2009;118(1):69-77.

9. Flannery JG, Farber DB, Bird AC, Bok D. Degenerative changes in a retina affected with autosomal dominant retinitis pigmentosa. Invest Ophthalmol Vis Sci. 1989;30:191-211.

10. Newman NM, Stevens RA, Heckenlively J. Nerve fiber layer loss in diseases of the outer retinal layer. Brit J Ophthalmol. 1987;71(1):21-26.

11. Oishi A, Otani A, Sasahara M, et al. Retinal nerve fiber layer thickness in patients with retinitis pigmentosa. Eye (Lond). 2009;23(3):561-566.

12. Sugita T, Kondo M, Piao CH, Ito Y, Terasaki H. Correlation between macular volume and focal macular electroretinogram in patients with retinitis pigmentosa. Invest Ophthalmol Vis Sci. 2008;49:3551-3558.

13. Wolsley CJ, Silvestri G, O'Neill J, Saunders KJ, Anderson RS. The association between multifocal electroretinograms and OCT retinal thickness in retinitis pigmentosa patients with good visual acuity. Eye (Lond). 2009;23(7):1524-1531.

14. Uggetti C, Egitto MG, Fazzi E, et al. Transsynaptic degeneration of lateral geniculate bodies in blind children: in vivo MR demonstration. Am J Neuroradiol. 1997;18(2):233-238.

15. Beatty RM, Sadun AA, Smith LEH, Vonsattel JP, Richardson Jr EP. Direct demonstration of transsynaptic degeneration in the human visual system: a comparison of retrograde and anterograde changes. J Neurol Neurosurg Psychiatry. 1982;45(2):143-146. 


\section{Publish your work in this journal}

Clinical Ophthalmology is an international, peer-reviewed journal covering all subspecialties within ophthalmology. Key topics include: Optometry; Visual science; Pharmacology and drug therapy in eye diseases; Basic Sciences; Primary and Secondary eye care; Patient Safety and Quality of Care Improvements. This journal is indexed on
PubMed Central and CAS, and is the official journal of The Society of Clinical Ophthalmology (SCO). The manuscript management system is completely online and includes a very quick and fair peer-review system, which is all easy to use. Visit http://www.dovepress.com/ testimonials.php to read real quotes from published authors. 